\title{
SPECTRUM OF RENAL ALLOGRAFT BIOPSY FINDINGS IN RENAL TRANSPLANT PATIENTS AT A TERTIARY CARE CENTER IN RAWALPINDI, PAKISTAN
}

\author{
Khurram Mansoor, Muhammad Osama, Muhammad Ishaque*, Sohail Sabir, Hafeez ud Din*, Muhammad Asif*, Haroon Sabir** \\ Armed Forces Institute of Urology/National University of Medical Sciences (NUMS) Rawalpindi Pakistan, *Armed Forces Institute of Pathology/National \\ University of Medical Sciences (NUMS) Rawalpindi Pakistan, **Pakistan Naval Ship Shifa Karachi Pakistan
}

\begin{abstract}
Objective: To evaluate outcome of diagnostic kidney biopsy in patients with renal allograft dysfunction at a tertiary care hospital.

Study Design: Retrospective observational study.

Place and Duration of Study: Armed Forces Institute of Urology, Rawalpindi, from Jan 2014 to Jan 2020.

Methodology: A consolidate registry review was carried to formulate this study. The registry data exists at our center containing information about the graft dysfunction (manifesting as proteinuria, deranged urea and creatinine or urine sediment abnormalities) and other major indications which warrant probing with biopsy. The histopathological diagnosis of these biopsies is confirmed from the nephro-pathology registry before finalization of diagnosis.

Results: A total of 94 diagnostic kidney biopsies were performed in patients with graft dysfunction. Out of 94 biopsies, 80 (85.1\%) patients were male while $14(14.9 \%)$ were female patients. The most frequent single cause for graft dysfunction was Cell Mediated Rejection (n 12, 24.5\%) followed by Interstitial Fibrosis and Tubular Atrophy/Acute Tubular Injury. The most common cause among the glomerulonephritis was Membranoproliferative Glomerulonephritis (n 3, 6.1\%) followed by others. The most common cause for mixed pathology remainedcell mediated rejection with Interstitial fibrosis and tubular atrophy (n 8, 17.8\%).

Conclusion: Cell mediated rejection is thecommonest pathology responsible for renal allograft dysfunction both as a single lesion as well as part of mixed pathology.
\end{abstract}

Keywords: Allograft dysfunction, Cell-mediated rejection, Renal biopsy.

This is an Open Access article distributed under the terms of the Creative Commons Attribution License (https://creativecommons.org/licenses/by-nc/4.0/), which permits unrestricted use, distribution, and reproduction in any medium, provided the original work is properly cited.

\section{INTRODUCTION}

Iversen and brun and alwall were the first clinicians who performed percutaneous renal biopsies in $1950 \mathrm{~s}^{1,2}$. In their procedure, patients were seated in upright position and the procedure achieved $>40 \%$ of tissue diagnosis. Kark and Muehrcke in 1954 modified the technique with a different set of needles and with patients in a prone position. As a result, $96 \%$ tissue diagnosis with no major complications were noted with the later technique.

Renal biopsy remains the cornerstone of investigations in renal allograft dysfunction, both in terms of diagnosis and management. Dysfunction of a kidney transplant often requires histological sampling by percutaneous ultrasound-guided core needle biopsy. Although the transplant kidney biopsy is more specialized than native kidney biopsy, the indications and complications are less well understood than the native kidney biopsy33.

The concept of protocol transplant biopsies has also evolved over time. Although the risks and benefits

Correspondence: Dr Khurram Mansoor, Consultant Nephrologist, Armed Forces Institute of Urology, Rawalpindi Pakistan

Received: 13 May 2020; revised received: 01 Jul 2020; accepted: 07 Jul 2020 of this procedure have long been debated but the sole benefit of achieving diagnosis in cases of subclinical rejection is of paramount importance in all such cases ${ }^{4}$. Further, favoring a proactive approach for doing diagnostic kidney biopsies in patients with renal allograft dysfunction is the fact that the estimated risk of serious complications in transplant kidneys is comparatively less than the native kidneys ${ }^{5,6}$. Further, in literature special importance has been laid on doing pre-emptive kidney biopsy in cadaveric donors. A very large sample sized study from Hungary have reported significant benefit in diagnosing problems like acute tubular necrosis, arteriosclerosis and chronic tubulointerstitial nephritis etc, in these kidneys on zero hour (just before the transplant surgery $)^{7}$.

Data suggests that the introduction of renal allograft biopsy has altered the diagnosis in $27-46 \%$ of patients and management in 38-83\%, even after the first year of transplant ${ }^{8}$. The discovery of C4d staining was a milestone in the diagnosis and management of renal allograft rejection ${ }^{9}$. The advanced techniques of tissue typing and cross matching are not easily and widely available in Pakistan and are also a financial burden for a majority of transplant candidates and their families. Under such circumstances where these 
tests are sometimes done with older and cost effective techniques the rejections could be picked up with early and sometimes pre-emptive biopsies. It is a recognized fact that graft survival rates and their causes vary in different transplant centers of Pakistan. This has actually acted as an impetus for conducting our study and to work out if the spectrum of causes in our institute is any different from others.

\section{METHODOLOGY}

This was a retrospective study conducted at Armed Forces Institute of Urology (ERC/ IRB no. UroAdm-Trg-1/IRB/2020/103), Rawalpindi, Pakistan. We assessed and interpreted the registry data of our institute dating from January 2014 to January 2020. Nonprobability consecutive sampling technique was used (WHO sample size calculator used with confidence interval $95 \%$, margin of error $5 \%$, population frequency adding acute and chronic rejection 34\%) to enroll 94 biopsy specimens after satisfying inclusion criteria (patients of either gender who underwent renal biopsy for graft dysfunction) while those having inade-quate specimen were excluded.

AFIU is one of the largest renal transplant centers in Northern Pakistan. Only first degree live-related renal transplants have been done in this center after seeking permission from federal Human Organ Transplant Authority. The transplanted patients if at all face graft dysfunction, undergo an early kidney biopsy. The transplant registry at our center contains clinical information about the patients who have graft dysfunction in detail. The histopathological diagnosis of these biopsies is obtained from nephro-pathology registry maintained in histopathology department of Armed Forces Institute of Pathology. The final diagnosis of graft dysfunction thus encompasses both the clinical and pathological parameters of our patients.

In this study, all patients first gave informed and written consent for undergoing the same standard operating procedure for doing diagnostic transplant kidney biopsy. Two cores from the renal graft were taken with Monopty (trucut) biopsy needle (18G) under real time ultrasound guidance. Light microscopy for histopathological assessment was done. All biopsies were reported by the same group of histopathology consultants (three in number). All patients were observed for 12 hours post-biopsy. Each patient underwent 2 consecutive urine routine examinations and two complete blood count evaluations 12 hours apart. They were closely watched for post-biopsy hematuria and any change in vital signs. Major post-biopsy complications if any were recorded for future consultation.

The data gathered, was analyzed by descriptive statistics such as mean and standard deviation (SD) for continuous variables and frequencies and percentages for qualitative variables. SPSS version 20 was used for statistical analysis.

\section{RESULTS}

The study was conducted from January 2014 to January 2020 and the number of renal transplant biopsies done over the above mentioned study period were 94. All included biopsies were adequate. Out of 94 biopsies, $80(85.1 \%)$ biopsies were from male patients while $14(14.9 \%)$ from female patients, as shown in figure.

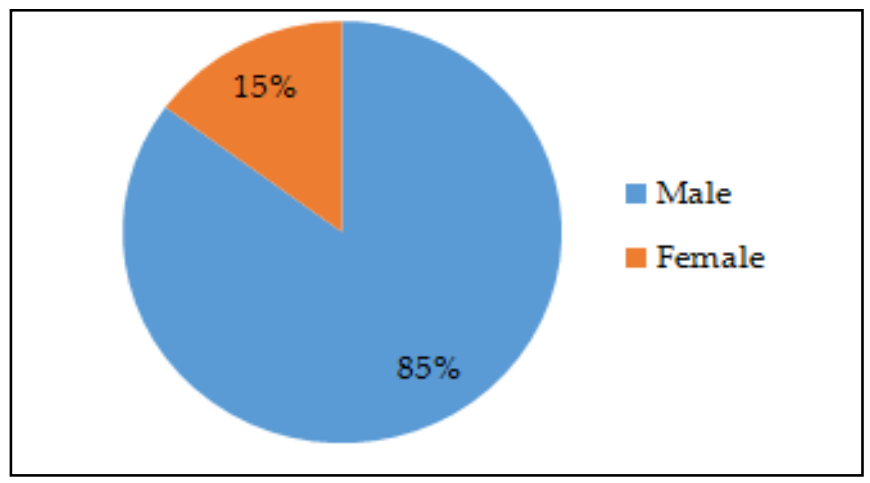

Figure: Pie-chart showing age distribution of all cases.

The mean age of all patients who underwent biopsies was $36.72 \pm 11.47$ years with range of $15-60$ years. The mean duration of biopsies from the date of transplant was $28.65 \pm 39.81$ months (range: 1-72 months). Around $50 \%$ of the biopsies were done within 24 months of transplant and out of these, $25 \%$ were done within first 7 months. The rest (50\%) of the biopsies were done 24 months post-transplant and out of these, $25 \%$ biopsies were done after 48 months of transplantation.

Out of 94 transplant biopsies, 49 (52.1\%) had single cause for graft dysfunction while 45 (47.9\%) had multiple causes for transplant dysfunction.

Among singular causes, the most common cause of renal transplant dysfunction was cell-mediated rejection (CMR) (n 12, 24.5\%), followed by Acute Tubular Injury (ATI) and Interstitial Fibrosis and Tubular Atrophy (IFTA) (n 8, 16.3\%). Membranoproliferative Glomerulonephritis (MPGN) was the most common form of glomerulonephritis (GN) in the category of single lesions (n3, 6.1\%). Three out of five patients with 
glomerulonephritis were found to have Membranoproliferative Glomerulonephritis, while Focal and Segmental Glomerulosclerosis (FSGS) and Membranous Glomerulonephritis (MN)were found in a single patient each. The single causes of allograft lesions are shown in table-I.

Table-I: Single pathological lesion on graft biopsies in patients with graft dysfunction $(n=94)$.

\begin{tabular}{l|c}
\hline Cause & $\mathbf{n ~ ( \% )}$ \\
\hline Cell mediated rejection & $12(24.5 \%)$ \\
\hline Anti-body mediated rejection & $3(6.1 \%)$ \\
\hline Glomerulonephritis & $5(10.2 \%)$ \\
\hline Interstitial fibrosis and tubular atrophy & $8(16.3 \%)$ \\
\hline Calcineurin inhibitor toxicity & $2(4.1 \%)$ \\
\hline Acute tubular injury & $10(20.4 \%)$ \\
\hline Borderline rejection & $4(8.2 \%)$ \\
\hline Renal vein thrombosis & $2(4.1 \%)$ \\
\hline Minor changes & $2(4.1 \%)$ \\
\hline BK virus nephropathy & $1(2.0 \%)$ \\
\hline
\end{tabular}

A total of $43(47.9 \%)$ biopsies showed more than one pathological lesion on microscopy. A breakdown of these combination of lesions is shown in table-II. The commonest combination was CMR and IFTA. with renal allograft dysfunction in Pakistan. The largest so far conducted Pakistani study, was published in 2012 by the Sindh Institute of Urology and Transplant by Kazi et al ${ }^{10}$. In terms of gender distribution, age and even histopathological lesions, their results are quite close to our study.

This study focuses on various causes of renal transplant dysfunction as detected on renal allograft biopsies in our center. All transplants done in our center were first degree relatives and $\mathrm{ABO}$ compatible. The male to female ratio was $4: 1$, which is almost the same as reported by Kazi et al (3:1) and even in other developing countries, including studies from neighboring countries like Nepal (by Aryal et al in 2012) and India (by Puntambekar et al in 2017 and Patil et al in 2018) ${ }^{10-13}$. In our study, the mean age of patients at the time of biopsy was $36.72 \pm 11.47$ years which is quite close to what Kazi et al found $(35.7 \pm 10.5)^{10}$. In developed countries, renal allograft rejection is higher in older aged donors ${ }^{14}$. Whereas, in majority of such studies, a younger recipient age group is associated with favorable outcome in terms of rejection ${ }^{14-16}$. Not only the donor and recipient age, but many other factors have also been implicated in various studies lea-

Table-II: Distribution of multiple pathological lesions on graft biopsies in patients with graft dysfunction (n=94).

\begin{tabular}{|c|c|}
\hline Cause & n (\%) \\
\hline Antibody mediated rejection + Calcineurin inhibitors toxicity & $1(2.2 \%)$ \\
\hline Antibody mediated rejection + Glomerulonephritis & $1(2.2 \%)$ \\
\hline Antibody mediated rejection + Interstitial fibrosis and tubular atrophy & $5(11.1 \%)$ \\
\hline Antibody mediated rejection + Interstitial fibrosis and tubular atrophy + Acute tubular injury & $1(2.2 \%)$ \\
\hline Antibody mediated rejection + Interstitial fibrosis and tubular atrophy + CNI toxicity & $1(2.2 \%)$ \\
\hline Antibody mediated rejection + Cell mediated rejection + Interstitial fibrosis and tubular atrophy & $1(2.2 \%)$ \\
\hline Antibody mediated rejection + Interstitial fibrosis and tubular atrophy + Glomerulonephritis & $1(2.2 \%)$ \\
\hline Borderline rejection + CNI toxicity + Interstitial fibrosis and tubular atrophy & $1(2.2 \%)$ \\
\hline Borderline rejection + Interstitial fibrosis and tubular atrophy & $1(2.2 \%)$ \\
\hline Cell mediated rejection + Antibody mediated rejection & $1(2.2 \%)$ \\
\hline Cell mediated rejection + Calcineurin inhibitor toxicity & $1(2.2 \%)$ \\
\hline Cell mediated rejection + Calcineurin inhibitor toxicity + Interstitial fibrosis and tubular atrophy & $3(6.7 \%)$ \\
\hline Cell mediated rejection + Interstitial fibrosis and tubular atrophy & $8(17.8 \%)$ \\
\hline Cell mediated rejection + Interstitial fibrosis and tubular atrophy + Acute tubular injury & $1(2.2 \%)$ \\
\hline Cell mediated rejection + Interstitial fibrosis and tubular atrophy + Glomerulonephritis & $1(2.2 \%)$ \\
\hline Glomerulonephritis + Acute tubular injury & $1(2.2 \%)$ \\
\hline Glomerulonephritis + Interstitial fibrosis and tubular atrophy & $6(13.3 \%)$ \\
\hline Glomerulonephritis + Interstitial fibrosis and tubular atrophy + Calcineurin inhibitor toxicity & $1(2.2 \%)$ \\
\hline Interstitial fibrosis and tubular atrophy + Acute tubular injury & $2(4.4 \%)$ \\
\hline Interstitial fibrosis and tubular atrophy + Pyelonephritis & $1(4.4 \%)$ \\
\hline Pigment cast nephropathy with interstitial nephritis & $1(2.2 \%)$ \\
\hline Polyoma virus nephropathy + Interstitial fibrosis and tubular atrophy & $2(4.4 \%)$ \\
\hline Transplant glomerulopathy + Interstitial fibrosis and tubular atrophy & $3(6.7 \%)$ \\
\hline
\end{tabular}

\section{DISCUSSION}

There was very little data available on the spectrum of renal allograft biopsy findings in patients ding to graft loss in patients with renal allografts ${ }^{17}$.

In our study, cell mediated rejection was the most common cause of renal allograft dysfunction followed 
by acute tubular injury and interstitial fibrosis and tubular atrophy respectively. The same pattern of distribution has been identified by Kazi et al as well but with slight variation as detailed below ${ }^{10}$.

Around $50 \%$ of the episodes of graft dysfunction occurred within 24 months post-transplant, among which, $25 \%$ occurred within 7 months of transplantation, while $25 \%$ episodesoccurred after 48 months.

Despite advancements in immune suppression and patient friendly protocols, the incidence of acute rejections still persists in renal transplant population. We have found acute rejections to be the commonest cause of graft dysfunction in our patients. Cell mediated rejection was present in $24.5 \%$ of causes. It was the most common pathology (both as single as well as part of mixed pathology). It was followed by Borderline rejection which was evident in $8.2 \%$ of the cases. Regarding cell mediated rejection, it is worth mentioning that it has even remained as the commonest cause in mixed pathology lesions in graft dysfunction as well. Antibody mediated rejection accounted for $6.1 \%$ of all the cases. The overall incidence of rejections in our study was only slightly higher than the previously published Pakistani study (24\% including all types of acute rejections) ${ }^{10}$. One explanation for this would be the presence of delayed graft dysfunction in some of our patients. Although they did not meet classical definition of delayed graft dysfunction i.e requiring dialysis in first week post-surgery rather they were slow to regain normal renal function otherwise. We are aware of the fact that there is a direct relationship between anyform of delayed graft dysfunction and rejection $^{18}$. This factor was not catered in the last published study so it is difficult to comment from their perspective $^{10}$. Rate of classical delayed graft function (patients requiring dialysis in first week after surgery) in our study was about $2 \%$ which matches with data from other centers permitting only live donor transplants ${ }^{16}$.

Most of our patients were inducted with Basiliximab in our institute. As per protocol at our center, all our transplant candidates falling in intermediate and low risk group are inducted with Basiliximab. Around $90 \%$ of our transplant recipients are usually form these two groups and thus receive Basiliximab. Rest of the patients, (from the high risk group) were inducted with Anti thymocyte globulinas a depleting agent. Although it has a protective effect on rejections but the main side effect of Anti thymocyte globulin is predisposition to various infections, which should be kept in mind in post-transplant period for ensuring ade- quate renal function. This situation further aggravates once these patients fail to comply with the follow up plan in the clinics and many times harbor sub-clinical infections.

Immunosuppression monitoring is widely available for calcineurin inhibitors, while no such monitoring is available for anti-proliferative drugs. Some physicians consider the number of cases of rejection as an indicator of adequacy of immunosuppression. The actual contribution of immunosuppression to renal transplant dysfunction is hard to estimate as the factor of infection secondary to immunosuppression is also a likely cause for graft dysfunction. That is why Kidney Disease Improving Global Outcome advocate the use of depleting agents for high-risk population only ${ }^{19}$. The rate of graft rejection varies in different parts of the world due to center-specific transplant protocols.

After rejections, second most common cause of transplant dysfunction was Acute tubular injury, which accounted for $20.4 \%$ (vs $24 \%$ by Kazi et al) ${ }^{10}$. This was followed by Interstitial fibrosis and tubular atrophy $(16.3 \%)$ and Calcineurin inhibitor toxicity $(4.1 \%)$. Whereas the calcineurin inhibitor toxicity as reported by Kazi et al was $11 \%{ }^{10}$. Since different tubular injuries mentioned above form a spectrum of pathology which often has overlapping features, therefore this disparity in distribution could be a difference of interpretation as well20.

Glomerulonephritis accounted for $10.2 \%$ cases. Membranoproliferative glomerulonephritis, was the most common type (6\%). It was difficult to differentiate between recurrent and de novo GN as native kidney disease is not known in most of the cases in our setup. Pigment cast nephropathy as a cause of Acute tubular injury was found in one of the biopsies. BK virus nephropathy constituted the least common isolated cause of graft dysfunction, i.e., $2 \%$.

When the biopsy findings discussed above were compared with the initial clinical presentation, we found that asymptomatic rise of serum creatinine was the most common patient presentation.

The limitations of our study include a retrospective study design, single center-based study and lacking the use of newer method of tissue staining for C4d, making it difficult to compare our study findings with older studies ${ }^{21}$. Likewise, the new and emerging biopsy assessment and interpretation techniques including molecular analysis techniques can also prove to be beneficial in confirming difficult diagnoses ${ }^{22}$. 
Despite the above, our study gives an excellent overview of biopsy-proven causes of graft dysfunction in our transplant population.

\section{CONCLUSION}

The gold standard investigation for the diagnosis of causes of renal allograft dysfunction is renal allograft biopsy. Asymptomatic rise of serum creatinine was the most common initial presentation of graft dysfunction. Cell mediated rejection remained the most common pathology both in single as well as mixed etiology for graft dysfunction in live related renal transplant patients.

\section{CONFLICT OF INTEREST}

This study has no conflict of interest to be declared by any author.

\section{REFERENCES}

1. Iversen P, Brun C. Aspiration biopsy of the kidney. J Am Soc Nephrol 1997; 8(11): 1778-86.

2. Alwall N. Aspiration biopsy of the kidney, including a report of a caseof amyloidosis diagnosed through aspiration biopsy of the kidney in 1944 and investigated at an autopsy in 1950. Acta Med Scand 1952; 143(6): 430-35.

3. Michael E. Reschen, Andrea Mazzella, Edward Sharples. A retrospective analysis of the utility and safety of kidney transplant biopsies by nephrology trainees and consultants. Ann Med Surg 2018; 28(2): 6-10

4. Fu M, Lim S, Jalalonmuhali M, Ng K, Ng P. Clinical significance of renal allograft protocol biopsies: a single tertiary center experience in malaysia. J Transplant 2019; 2019: 9153875.

5. Whittier W, Gashti C, Saltzberg S, Korbet S. Comparison of native and transplant kidney biopsies: diagnostic yield and complications. Clin Kidney J 2018; 11(5): 616-22.

6. Trajceska L, Severova-Andreevska G, Dzekova-Vidimliski P, Nikolov I, Selim G, Spasovski G, et al. Complications and risks of percutaneous renal biopsy. J Med Sci 2019; 7(6): 992-95

7. Szakaly P, Kalmar-Nagy K, Praksch D, Vas T, Varga A. More than twenty years' experience with "zero-hour" transplant kidney biopsy. Clin Surg 2018; 10(3): 2153.

8. El-Zoghby ZM, Stegall MD, Lager DJ, Kremers WK, Amer H, Gloor JM, et al. Identifying specific causes of kidney allograft loss. Am J Transplant 2009; 9(3): 527-35.
9. Ranjan P, Nada R, Jha V, Sakhuja V, Joshi K. The role of C4d immunostaining in the evaluationof the causes of renal allograft dysfunction. Nephrol Dial Transplant 2008; 23(5): 1735-41.

10. Kazi JI, Mubarak M. Biopsy findings in renalallograft dysfunction in a live related renal transplant program. J Transplant Technol Res 2012; 2(1): 108.

11. Aryal G, Shah DS. Histopathological evaluation of renal allograft biopsies in Nepal: Interpretation and significance. J Pathol Nepal 2012; 2(3): 172-79.

12. Puntambekar A, Parameswaran S, Nachiappa R. Evaluation of clinico-pathological spectrum in renal allograft biopsies at JIPMER. J Kidney 2017; 3: 3.

13. Patil MR, Divyaveer SS, Mahajan C, Choudhury AR, Dasgupta S, Sarkar D, et al. Spectrum of renal allograft biopsy: a five-year experience at a tertiary care center of eastern India. Saudi J Kidney Dis Transpl 2018; 29(4): 930-38.

14. Kostakis ID, Moris DN, Barlas A, Bokos I, Darema M, Theodoropoulou $\mathrm{E}$, et al. Impact of donor and recipient age difference on long-term allograft survival after living donor renal transplantation: Analysis of 478 cases. Clin Transplant 2013; 27(6): 838-43.

15. Joosten SA, Sijpkens YW, van Kooten C, Paul LC. Chronic renal allograft rejection: Pathophysiologic considerations. Kidney Int 2005; 68(1): 1-3.

16. United states renal data system (USRDS). 2011 USRDS Annual Data Report on Transplantation. [Internet] Available at: https:// www.usrds.org/2011/view/v1_00a_intro.asp

17. Ndemera H, Bhengu B. Factors contributing to kidney allograft loss and associated consequences among post kidney transplantation patients. Health Sci J 2017; 11(3): 504.

18. Wu WK, Famure O, Li Y, Kim SJ. Delayed graft function and the risk of acute rejection in the modern era of kidney transplantation. Kidney Intl 2015; 88 (4): 851-58.

19. Kidney Disease improving Global Outcome. KDIGO clinical practice guideline for the care of Kidney Transplant Recipient. [Internet] Available from: https://kdigo.org/wp-content/ uploads/2017/02/KDIGO-2009-Transplant-Recipient-GuidelineEnglish.pdf.

20. Nankivell BJ, Shingde M, Keung KL, Fung C, Borrows R, $\mathrm{O}^{\prime}$ Connell $\mathrm{P}$, et al. The causes, significance and consequences of inflammatory fibrosis in kidney transplantation: The Banff i-IFTA lesion. Am J Transplant 2018; 18(2): 364-76.

21. Troxell ML, Weintraub LA, Higgins JP, Kambham N. Comparison of C4d immunostaining methods in renal allograft biopsies. Clin J Am Soc Nephrol 2006; 1(3): 583-91.

22. Moreso F, Sellarès J, Soler MJ, Serón D. Transcriptome analysis in renal transplant biopsies not fulfilling rejection criteria. Int J Mol Sci 2020; 21(6): 2245. 